\title{
The Fuzzy Line Between Among and Surround
}

\author{
Pascal Matsakis ${ }^{1}$, Serge Andréfouët ${ }^{2}$ \\ ${ }^{1}$ University of Missouri-Columbia, Department of Computer Engineering and Computer Science \\ 201 Engineering Building West, Columbia, MO 65211, USA \\ pmatsakis@cecs.missouri.edu \\ ${ }^{2}$ University of South Florida, College of Marine Science, Remote Sensing and Biological Oceanography \\ 140, 7th Av. South, St Petersburg, FL 33701, USA \\ serge@ carbon.marine.usf.edu
}

\begin{abstract}
Spatial relationships among image objects play a vital role in countless domains of computer vision (e.g., pattern recognition, image understanding, scene description). Some have received considerable attention the last few years (e.g., "to the right of," "above," "to the left of" and "below"), but others have not been the subject of as much investigation. In this paper, we design consistent fuzzy models of three important spatial relationships: "surrounded by," "between" and "among." These models are based on the histogram of forces, which represents the relative position between two objects. Here, force histograms are assimilated to fuzzy sets and processed through their $\alpha$-cuts. Our goal is to extend the capabilities of a fuzzy system for linguistic scene description introduced in an earlier paper.
\end{abstract}

\section{INTRODUCTION}

Spatial relationships among image objects play a vital role in countless domains of computer vision. Freeman tried to draw up an exhaustive list of "primitive" relationships and came up with 13 names (such as "above", "near", "between", "inside", etc.) [1]. He also proposed the use of fuzzy relations, because "all or nothing" standard mathematical relations are clearly not suited for models of spatial relationships. Freeman's ideas were widely adopted. However, 2D objects were often assimilated to very elementary entities such as a point (centroid) or a (bounding) rectangle. The procedure is practical, but yields poor models because of the loss of a lot of morphological information. Krishnapuram et al [2] and Miyajima and Ralescu [3] tackled this problem in parallel and came up with similar propositions. They developed the idea that the relative position between two objects can have a representation of its own and can thus be described in terms other than spatial relationships. However, the representation set out in [3] shows several weaknesses (e.g., requirement for raster data, long processing times, anisotropy). In [4][5], Matsakis and Wendling introduced the histogram of forces. It proved to be a powerful tool, studied for numerous applications (e.g., pattern recognition [6], scene matching [7], linguistic scene description [8], spatial databases [9]).

The histogram of forces lends itself, with great flexibility, to fuzzy definitions of directional spatial relations (such as "to the right of", "in front of", etc.) [8]. In this paper, we consider its use for the modeling of other important relationships: "surrounded by," "between" and "among." They are represented by fuzzy relations: $s, b$ and $a$. Although the preposition "between" usually denotes a ternary relation, its model $b$ is binary, as are $s$ and $a$. For instance, the degree of truth of the proposition " $A$ is between $B_{1}$ and $B_{2}$ " is $b\left(A, B_{1} \cup B_{2}\right)$, and the degree of truth of " $A$ is among $B_{1}, B_{2}, B_{3}$ and $B_{4}$ " is $a\left(A, \cup B_{i}\right)$. Contrary to "between," the term "surrounded by" does not belong to the list of primitive names for spatial relations drawn up by Freeman in [1]. However, it can be related to the element that Freeman refers to as "inside." The relation "surround" then corresponds to "outside." It is the semantic inverse of "surrounded by," i.e., the propositions " $A$ is surrounded by $B_{1}, B_{2}$ and $B_{3}$ " and " $B_{l}$, $B_{2}$ and $B_{3}$ surround $A$ " are equivalent. The term "among" does not belong to Freeman's list either. We call "among" a spatial relationship similar to "between." The main difference is that "between" usually involves three objects $\left(A\right.$, and $B_{1}$ and $\left.B_{2}\right)$ whereas "among" involves four objects or more $(A$, and at least three other objects).

Fuzzy definitions of "surrounded by," "between" and "among" are proposed in Section III, and tested on real data in Section IV. Concluding remarks are given in Section V. Section II covers some existing models and briefly presents the notion of the histogram of forces.

\section{BACKGROUND}

\section{A. The Histogram of Forces}

The relative position of a $2 \mathrm{D}$ object $A$ with regard to another object $B$ can be represented by a function $F^{A B}$ from $\mathbb{R}$ into $\mathbb{R}_{+}$called the histogram of forces associated with $(A, B)$ via $F$, or the $F$-histogram associated with $(A, B)$. For any direction $\theta$, the value $F^{A B}(\theta)$ is the scalar resultant of elementary forces. These forces are exerted by the points of $A$ on those of $B$, and each tends to move $B$ in direction $\theta$ (Fig. 1). Actually, the letter $F$ denotes a numerical function. Consider any real number $r$. If the elementary forces are in inverse ratio to $d^{r}$, where $d$ represents the distance between the points considered, then $F$ is denoted by $F_{r}$. The $F_{0}$-histogram (histogram of constant forces) and the $F_{2}$-histogram (histogram of gravitational forces) have very different and very interesting characteristics. The former provides a global view of the situation. It gives equal consideration to both the object's closest and farthest parts, whereas the $F_{2}$-histogram focuses on the closest parts. 


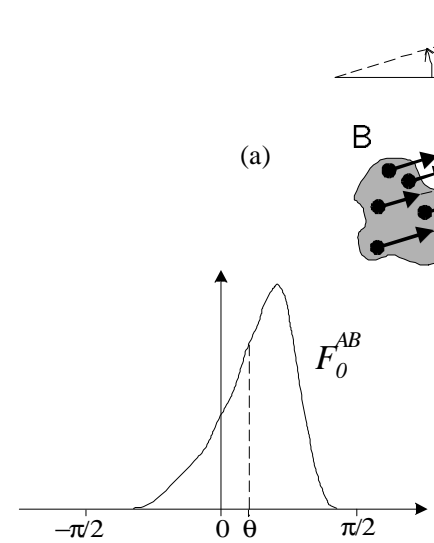

(b) (c)

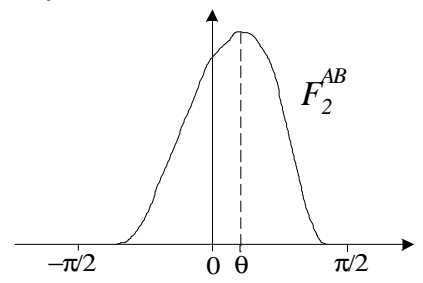

Fig. 1. Force histograms. (a) $F^{A B}(\theta)$ is the scalar resultant of forces (black arrows). Each one tends to move $B$ in direction $\theta$. (b) The histogram of constant forces associated with $(A, B)$. It represents the position of $A$ relative to $B$. (c) The histogram of gravitational forces associated with $(A, B)$. It is another representation of the relative position between $A$ and $B$.

The histogram of forces encapsulates structural information about the objects as well as information about their spatial relationships. It offers solid theoretical guarantees and nice geometric properties, ensures fast and efficient processing of vector data as well as of raster data, and enables the handling of fuzzy objects as well as crisp objects, intersecting objects as well as disjoint objects, and unbounded objects as well as bounded objects. Force histogram computation benefits from the power of integral calculus, is highly parallelizable, and utilizes a well-known algorithm that is commonly circuit coded in visualization systems. Details can be found in [4][5][7][8].

\section{B. Existing Spatial Models}

Spatial relationships like "surrounded by," "between" and "among" play an important role in the interpretation of a scene, and several methods of assessing these relationships have been proposed. There are two main approaches. The first one is based on such rules as $\left(\mathrm{R}_{1}\right)$ and $\left(\mathrm{R}_{2}\right)$ [10][11]:

( $\left.\mathrm{R}_{1}\right)$ IF $B_{1}$ is to the right of $A$ AND $B_{2}$ is to the left of $A$ THEN $A$ is between $B_{1}$ and $B_{2}$

$\left(\mathrm{R}_{2}\right)$ IF $B$ is to the right of $A$ AND $B$ is above $A$ AND

$B$ is to the left of $A$ AND $B$ is below $A$ THEN $A$ is surrounded by $B$

The main weakness of this approach comes from the fact that it is very difficult to express the necessary conditions for a spatial configuration to occur (instead of sufficient conditions). In the case of Fig. 2(a) for instance, the proposition " $A$ is between $B_{1}$ and $B_{2}$ " cannot be assessed using $\left(\mathrm{R}_{1}\right)$. Only a low (and useless) lower bound of its degree of truth would be obtained. Moreover, a rule like $\left(\mathrm{R}_{2}\right)$ assumes that an object can be in many directions with respect to another. This way of modeling directional relationships is questionable: generally, people do not combine more than two spatial preposi- tions when translating visual information into natural language descriptions [12][13]. Lastly, one should be aware of the implicit assumptions on the objects. $\left(\mathrm{R}_{2}\right)$, for instance, should be applied only if $B$ does not intersect the convex hull of $A$, i.e., if it is known that $A$ does not surround $B$ at all. Indeed, the directional relationships are tied by the semantic inverse principle [1] (e.g., $A$ is to the left of $B$ as much as $B$ is to the right of $A$ ). Therefore, without constraints on the objects, there is no way to know which object surrounds (or includes!) the other. This is illustrated by Figs. 2(b)(c).

In the second approach, the considered relationships are not derived from other "even-more-primitive" relationships. Instead they are assessed directly. Most examples can be found in the modeling of "surrounded by" and are based on the computation of a histogram of angles. For any pixel $P$ of $A$, let $\theta_{P}$ be the angle made by the two tangents from $P$ to $B$ as in Fig. 3. To each element $\theta$ of $[0,2 \pi]$, the histogram associates the number of pixels $P$ such that the angle $\theta_{P}$ is equal to $\theta$. In [14], the degree of truth for " $A$ is surrounded by $B$ " is produced by a multilayer perceptron fed by the histogram values. Other authors resort to a decreasing membership function $\mu$ from $[0,2 \pi]$ into $[0,1]$ such that $\mu(\theta)$ is 1 if $\theta$ is 0 and is 0 if $\theta$ is greater than $\pi$. In [11] for instance, the histogram of angles is assimilated to a fuzzy set and matched to $\mu$. In [2], it is used to compute the aggregated value of the $\mu\left(\theta_{P}\right)$ when $P$ describes $A$. The spatial models above all derive from Rosenfeld's visual surroundedness [15]. They assume that the object $B$ is connected and does not intersect $A$. They are not extremely robust since the results are not sensitive to the thickness of the surrounding object, only to tangency points. Moreover, they cannot handle vector data, and are computationally expensive.
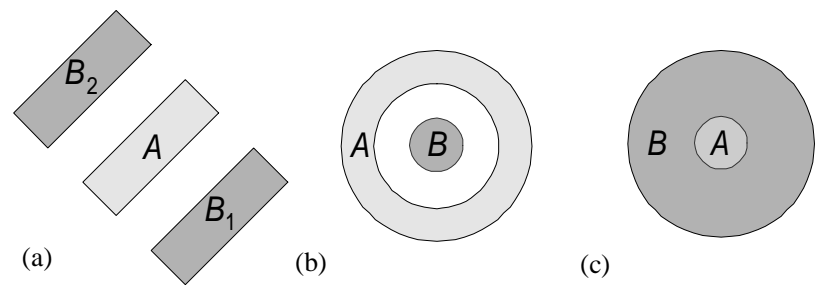

(b)

(c)

Fig. 2. (a) " $B_{1}$ is to the right of $A$ " and " $B_{2}$ is to the left of $A$ " are true to a certain extent only. Most fuzzy models [16] would produce degrees of truth lower than 0.5 . However, $A$ is clearly between $B_{1}$ and $B_{2}$. In (b) as in (c), the same fuzzy models would assess $B$ to be somewhat above, below, to the right and to the left of $A$ as well. It does not mean that $A$ is surrounded by $B$. In (b), $A$ surrounds $B$. In (c), $A$ is included in $B$

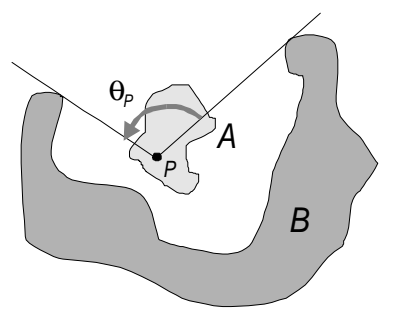

Fig. 3. Angle histogram computation for the modeling of "surrounded by." 


\section{NEW SPATIAL MODELS}

In this section, two objects, $A$ and $B$, are considered. The normalized histogram $F_{r}^{B A} / \max _{\theta} F_{r}^{B A}(\theta)$ is denoted by $H$ and assimilated to a fuzzy set (Fig. 4(a)). The symbol $H^{\alpha}$ denotes the support of $H$ if $\alpha$ is 0 , and the cut of level $\alpha$ if $\alpha$ belongs to the interval $(0,1]$ (Fig. 4(b)). For any $\alpha$, the set $H^{\alpha}$ is represented by a polar diagram (Fig. 4(c)). Imagine that $A$ and $B$ are the objects of Fig. 4(d) (B is composed of four connected components). When the radius of $A$ and the thickness of $B$ both tend towards 0 , the normalized histogram $H$ becomes crisp, and the polar representation of any $H^{\alpha}$ becomes exactly like Fig. 4(c), i.e., it merges with the configuration in the object space.

With any set $H^{\alpha}$ we associate $2 q$ angles: $z_{1}, z_{2}, \ldots, z_{q}$ ("z" as in zero) and $o_{1}, o_{2}, \ldots, o_{q}$ ("o" as in one). All these angles belong to the interval $[0,2 \pi]$. Moreover: $z_{l} \geq z_{2} \geq \ldots \geq z_{q}$ and $o_{I} \geq o_{2} \geq \ldots \geq o_{q}$. The value $q$ is the number of arcs in the polar representation of $H^{\alpha}$. This is illustrated by Fig. 4(e). When $H^{\alpha}$ is equal to the referential set (i.e., the set of real numbers), $q=1$ and $z_{l}=0$ and $o_{l}=2 \pi$ (case of Fig. 5(a)). If $q$ is greater than 1, two other angles $y_{1}$ and $y_{2}$ can be defined (Fig. 4(e)). We have: $y_{1} \geq z_{1}+z_{2}$ and $y_{2} \geq z_{1}+z_{2}$.

\section{A. "Surrounded by" $\alpha$-Cuts}

The degree to which the set $H^{\alpha}$ describes a "surrounded" situation is denoted by $s^{\alpha}(A, B)$. It is a real number that belongs to the interval $[0,1]$. We propose the simple expression below, where $k$ denotes some positive value (in our experiments, $k=1$ ). The highest possible degree, 1 , is reached when $z_{1}$ is 0 (see Fig. 5(a)). The higher $z_{1}$, the lower $s^{\alpha}(A, B)$ (see Figs. 5(b)(d)). Note that $s^{\alpha}(A, B)$ does not depend on the number of arcs that are around the center point in the polar representation of $H^{\alpha}$ (as far as $z_{1}$ remains the same).

$$
s^{\alpha}(A, B)=\max \left(0,1-k \frac{z_{1}}{\pi}\right)
$$

\section{B. "Between" $\alpha$-Cuts}

The degree to which the set $H^{\alpha}$ describes a "between" situation is denoted by $b^{\alpha}(A, B)$. We consider that the relationship should not apply when $q$ is 1 , and that the highest values should be reached when $q$ is 2 (i.e., when exactly two arcs are around the center point in the polar representation of $\left.H^{\alpha}\right)$. However, $z_{1}$ and $z_{2}$ should be close to each other, i.e., the ratio $z_{2} / z_{1}$ should be high (Fig. 6(a)). There is nothing preventing $z_{1}$ and $z_{2}$ from being small (Fig. 6(b)), but $\min \left(y_{1}, y_{2}\right)$ should not be too small (Fig. 6(c)). The situation is not as ideal when $q$ is greater than 2 . In accordance with Figs. 6(d)(e)(f), we consider that $z_{3}$ should be small compared to $z_{1}$ and $z_{2}$, i.e., the higher $z_{3} / z_{2}$, the lower $b^{\alpha}(A, B)$. In conclusion, we propose the definition below, where $k, k^{\prime}$ and $k^{\prime \prime}$ denote three positive real numbers (in our experiments, $k=1, k^{\prime}=1$ and $k^{\prime \prime}=2$ ).

$$
q=1 \Rightarrow b^{\alpha}(A, B)=0
$$

$$
\begin{aligned}
& q=2 \Rightarrow b^{\alpha}(A, B)=\min \left(1, k \frac{z_{2}}{z_{1}}, k^{\prime} \frac{y_{1}}{\pi}, k^{\prime} \frac{y_{2}}{\pi}\right) \\
& q>2 \Rightarrow b^{\alpha}(A, B)=\min \left(1, k \frac{z_{2}}{z_{1}}, k^{\prime} \frac{y_{1}}{\pi}, k^{\prime} \frac{y_{2}}{\pi}, \max \left(0,1-k^{\prime} \frac{z_{3}}{z_{2}}\right)\right) \\
& \text { C. “Among” } \alpha \text {-Cuts }
\end{aligned}
$$

The degree to which the set $H^{\alpha}$ describes an "among" situation is denoted by $a^{\alpha}(A, B)$. When $q$ is lower than 3 , we consider that the relationship should not apply. When $q$ is greater than or equal to 3 , we expect the arcs in the polar representation of $H^{\alpha}$ to be comparable in size and evenly distributed around the center point. The best case is illustrated by Figs. 7(a)(d), in which $z_{l}=z_{q}$ and $o_{I}=o_{q}$, i.e., $z_{l}=\left(\Sigma_{i} z_{i}\right) / q$ and $o_{l}=\left(\Sigma_{i} o_{i}\right) / q$. The worst cases are illustrated by Figs. 7(b)(e), in which either $z_{1} \gg z_{2}$ or $o_{1} \gg o_{2}$, i.e., $z_{1} \approx \Sigma_{i} z_{i}$ or $o_{l} \approx \Sigma_{i} o_{i}$. Hence the proposed definition below:

(a)

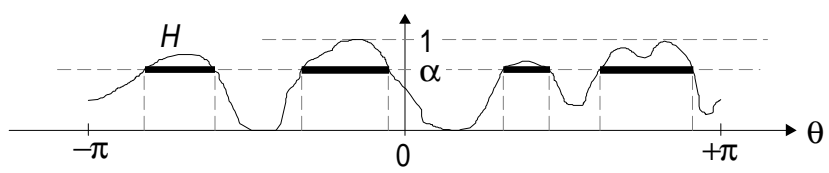

(b)

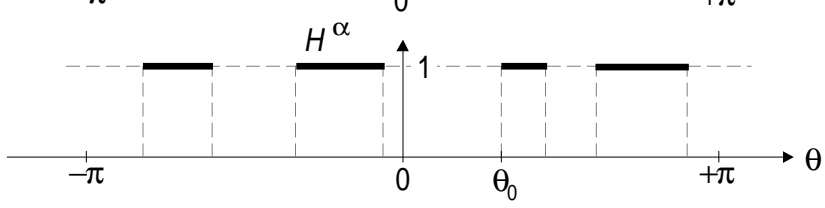

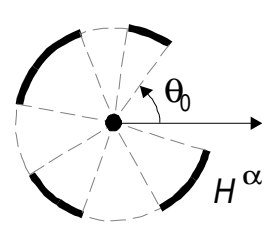

(c)

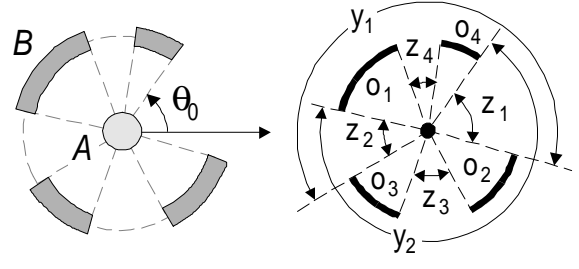

(d)

(e)
Fig. 4. (a) The normalized force histogram $H$ is assimilated to a fuzzy set. (b) $H^{\alpha}$ is the cut of level $\alpha$. (c) Polar representation of $H^{\alpha}$. We would get a very similar diagram if the objects $A$ and $B$ were as in (d). (e) Angles associated with $H^{\alpha}$

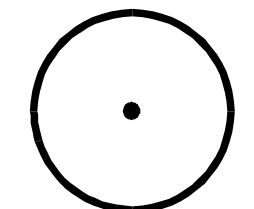

(a)

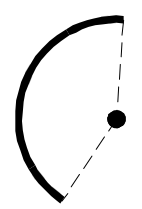

(d)

Fig. 5. Degree to which $H^{\alpha}$ describes a "surrounded" situation. (a) $s^{\alpha}(A, B)=1$. (b) $s^{\alpha}(A, B) \approx 0.5$. (c) $s^{\alpha}(A, B) \approx 0.5$. (d) $s^{\alpha}(A, B)=0$. (e) $s^{\alpha}(A, B) \approx 0.9$. (f) $s^{\alpha}(A, B) \approx 0.8$. (b)

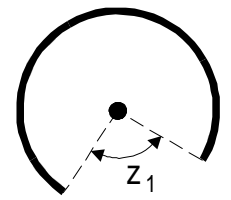

(c)
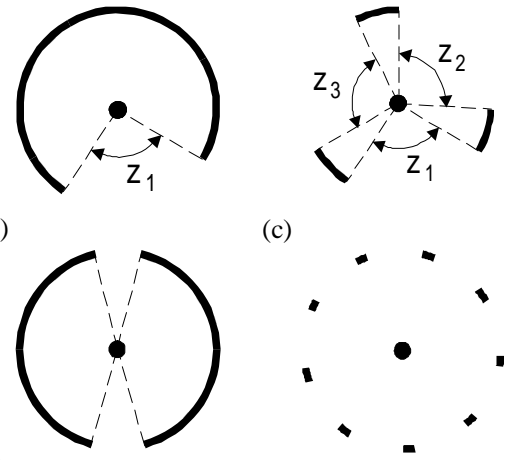


$$
\begin{aligned}
& q<3 \Rightarrow a^{\alpha}(A, B)=0 \\
& q \geq 3 \Rightarrow a^{\alpha}(A, B)=\min \left(\frac{\Sigma_{i} z_{i}-z_{1}}{\Sigma_{i} z_{i}-\left(\Sigma_{i} z_{i}\right) / q}, \frac{\Sigma_{i} o_{i}-o_{1}}{\Sigma_{i} o_{i}-\left(\Sigma_{i} o_{i}\right) / q}\right)
\end{aligned}
$$

\section{Wrapping Computation Scheme}

The spatial relationships "surrounded by," "between" and "among" are modeled by fuzzy binary relations: $s, b$ and $a$. One might consider that the histogram values are not really useful, and that the most important is the knowledge of the directions in which forces appear-whatever the type and the amplitude of these forces. The relations $s, b$ and $a$ could, therefore, be defined as follows: $s(A, B)=s^{0}(A, B), b(A, B)=b^{0}(A, B)$ and $a(A, B)=a^{0}(A, B)$. The method, however, is drastic. Moreover, it is not extremely robust. Although the values $s^{\alpha}(A, B), b^{\alpha}(A, B)$ and $a^{\alpha}(A, B)$ vary in a continuous manner when an arc or a gap in the polar representation of $H^{\alpha}$ extends gradually at one of its ends, continuity is generally disrupted when an arc stretches to merge with its neighbor or shrinks to disappear.

All $H^{\alpha}$ sets should therefore be considered. In practice, the fuzzy set $H$ can be represented by a finite number of $\alpha$ cuts: $H^{\alpha_{1}}, H^{\alpha_{2}}, \ldots, H^{\alpha_{n}}$. The values $\alpha_{1}, \alpha_{2}, \ldots, \alpha_{n}$ are such that: $\alpha_{1}=1>\alpha_{2}>\ldots>\alpha_{n}>\alpha_{n+1}=0$ and $H^{\alpha_{l}} \subset H^{\alpha_{2}} \subset \ldots \subset H^{\alpha_{n}}=H^{0}$.

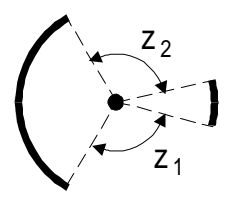

(a)

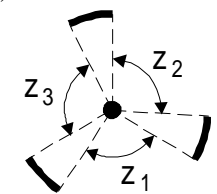

(d)

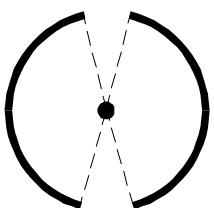

(b)

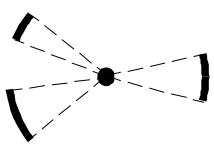

(e)

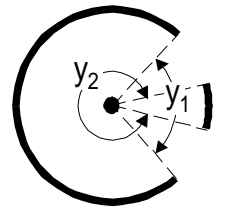

(c)

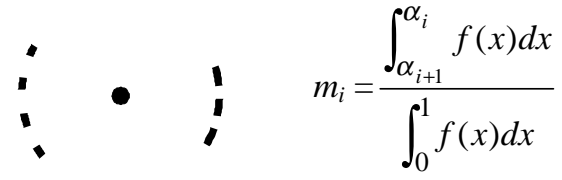

(f)

Fig. 6. Degree to which $H^{\alpha}$ describes a "between" situation.

(a) $b^{\alpha}(A, B)=1$. (b) $b^{\alpha}(A, B)=1$. (c) $b^{\alpha}(A, B) \approx 0.4$.

(d) $b^{\alpha}(A, B)=0$. (e) $b^{\alpha}(A, B) \approx 0.5$. (f) $b^{\alpha}(A, B) \approx 0.8$.

(a)
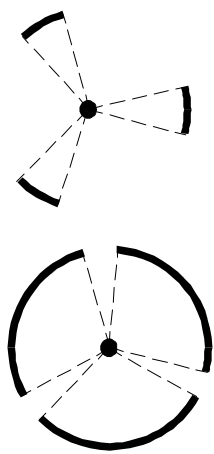

(d) (b)
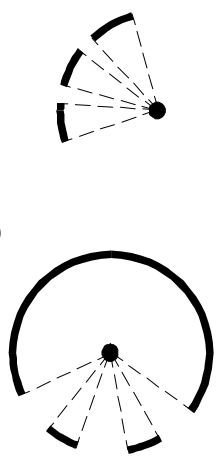

(e)

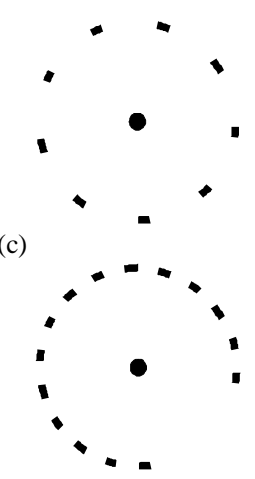

(f)
Fig. 7. Degree to which $H^{\alpha}$ describes an "among" situation. (a) $a^{\alpha}(A, B)=1$. (b) $a^{\alpha}(A, B) \approx 0.1$. (c) $a^{\alpha}(A, B)=1$. (d) $a^{\alpha}(A, B)=1$. (e) $a^{\alpha}(A, B) \approx 0.2$. (f) $a^{\alpha}(A, B) \approx 0.8$.
The degrees of truth $s(A, B), b(A, B)$ and $a(A, B)$ can then be defined as follows, using the general computation scheme proposed by Dubois and Jaulent in [17]:

$$
\begin{aligned}
& \qquad \begin{array}{l}
s(A, B)=\sum_{i} m_{i} s^{\alpha_{i}}(A, B), \\
b(A, B)=\sum_{i} m_{i} b^{\alpha_{i}}(A, B) \\
\text { and } a(A, B)=\sum_{i} m_{i} a^{\alpha_{i}}(A, B), \\
\text { where } m_{i}=\alpha_{i}-\alpha_{i+1} .
\end{array}
\end{aligned}
$$

The results presented in [17] rely on Shafer's theory of belief functions [18] and its links with fuzzy set and possibility theory [19]. Such links make statistical interpretation of membership functions possible [17]. For instance, the spatial relationship "between" measured on $(A, B)$ yields the following probability distribution:

$$
\begin{aligned}
p_{b} \mid[0,1] & \rightarrow[0,1] \\
x & \mapsto \Sigma_{i} m_{i} \delta\left(x, b^{\alpha_{i}}(A, B)\right)
\end{aligned}
$$

where $\delta\left(x, b^{\alpha_{i}}(A, B)\right)$ is 1 if $x$ is equal to $b^{\alpha_{i}}(A, B)$ and is 0 otherwise. The degree $b(A, B)$ as stated in Equation (2) is simply the corresponding expected value. Instead of this single number, a fuzzy interval could also be obtained by transforming $p_{b}$ into a possibility distribution [17]. In this paper, Equation (4) will be replaced by Equation (5) below, where $f$ denotes a continuous non-increasing function from $[0,1]$ to $\mathbb{R}_{+}$. If $f(x)$ is 1 , then (4) and (5) are equivalent. Four examples of functions $f$ are given by Fig. 8 .

$$
m_{i}=\frac{\int_{\alpha_{i+1}}^{\alpha_{i}} f(x) d x}{\int_{0}^{1} f(x) d x}
$$

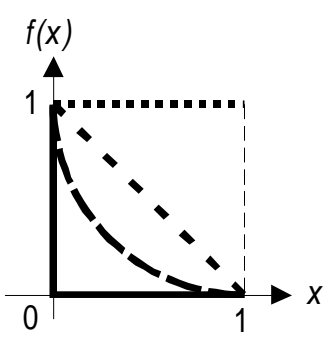

Fig. 8. Examples of functions $f$ for the definition of basic probability assignments.
The L-shaped function corresponds to the case where the sets $H^{\alpha}$ are all ignored but $H^{0}$. As mentioned above, the support $H^{0}$ seems to be the most appropriate set for describing the relationships between the objects $A$ and $B$ (whereas, paradoxically, the core $H^{l}$ seems to be the less significant). Considering different types of force, and other basic probability assignments than the ones defined by (4), makes it possible to design spatial models that are more or less sensitive to the variability of the forces at work, i.e., to the relative thickness of the objects and to the relative distance between them. This is illustrated by Fig. 9 and Table I. When relying on constant forces $\left(F_{0}\right)$, the "between" models do not care about the increasing distance in situations 4,5 and 6 . However, they are very sensitive to the length of the right rectangle in situations 1,2 and 3 . This sensitivity can be controlled by $f$. With gravitational forces $\left(F_{2}\right)$, it is just the opposite. The models pay special attention to relative distance and much less to 


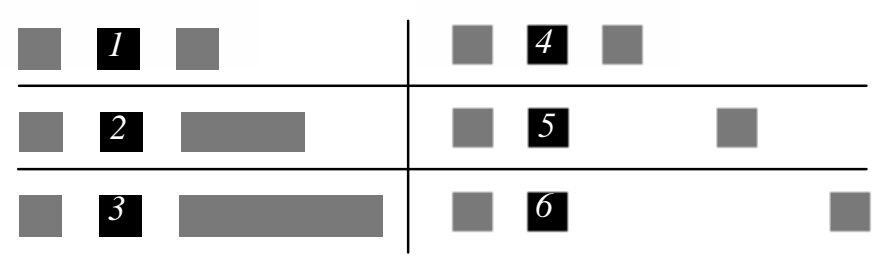

Fig. 9. Different "between" situations.

$A$ is the black square, $B$ is the union of the two gray rectangles.

TABLE I

THE VALUES OF $b(A, B)$ FOR THE SITUATIONS IN FIG. 9 DEPEND ON THE FORCE HISTOGRAM AND ON THE BASIC PROBABILITY ASSIGNMENT

\begin{tabular}{|c|c|c|c|c|c|c|c|c|}
\hline & \multicolumn{4}{|c|}{$F_{0}$} & \multicolumn{4}{|c|}{$F_{2}$} \\
\hline & & & & & & & & \\
\hline 1 & 1.00 & 1.00 & 1.00 & 1.00 & 1.00 & 1.00 & 1.00 & 1.00 \\
\hline 2 & 1.00 & 0.77 & 0.58 & 0.35 & 1.00 & 0.96 & 0.87 & 0.64 \\
\hline 3 & 1.00 & 0.57 & 0.38 & 0.21 & 1.00 & 0.93 & 0.81 & 0.56 \\
\hline 4 & 1.00 & 1.00 & 1.00 & 1.00 & 1.00 & 1.00 & 1.00 & 1.00 \\
\hline 5 & 1.00 & 1.00 & 1.00 & 1.00 & 1.00 & 0.42 & 0.26 & 0.14 \\
\hline 6 & 1.00 & 1.00 & 1.00 & 1.00 & 1.00 & 0.19 & 0.10 & 0.05 \\
\hline
\end{tabular}

relative thickness. For our experiments, in Section IV, we used $F_{2}$-histograms and the basic probability assignments defined by the arc-shaped function. They gave the best intuitive results (according to our own perception).

\section{EXPERIMENTS}

We considered two LADAR (Laser Radar) images provided by the Naval Air Warfare Center (Fig. 10). Both were used in previous works to test different fuzzy systems for automatic target recognition [20] and linguistic scene description [8]. The system presented in [8] relies on the computation of force histograms. It outputs descriptions that make use of spatial prepositions related to directional relationships. For instance, the object $A$ in Fig. 10(b) is found to be "perfectly to the right of $B_{1}$," "below-right of $B_{2}$," etc. The histograms computed are $F^{A B_{1}}, F^{A B_{2}}$, etc. Consider the group of buildings 4,5 and 6 in Fig. 10(d). Relative to that group, the tower $l$ is "to the left but a little above," the tower 2 is "perfectly to the left," and the storehouse 3 is "perfectly above but slightly shifted to the left." Now, consider Fig. 10(b) again. If the system was asked to describe the position of $A$ with respect to $\cup B_{i}$, it would have to generate $F^{\left(\cup B_{i}\right) A}$. This is an easy task, because $F^{\left(\cup B_{i}\right) A}=\Sigma_{i} F^{B_{i} A}$ and: $\forall \theta \in \mathbb{R}, F^{B A}(\theta)=F^{A B}(\theta-\pi)$ [7]. The first equation comes from the fact that forces are additive and $\cap B_{i}$ is empty. Knowing $F^{\left(\cup B_{i}\right) A}$, the system would then be able to output the requested linguistic description. However, in this particular case, it would come to the conclusion that none of the directional relationships are relevant, and would display the message "???????" We now have the ability to extend the system.

Fig. 11 shows ten configurations extracted from Figs. 10 (b)(d). For each configuration, the degrees of truth $s(A, B)$, $b(A, B)$ and $a(A, B)$ have been computed (see Table II). The highest degree, if greater than some threshold $(0.5)$, indicates
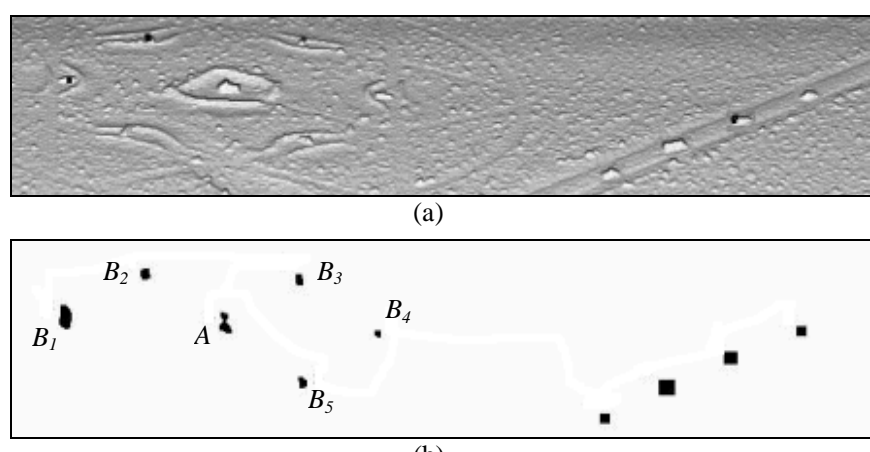

(b)

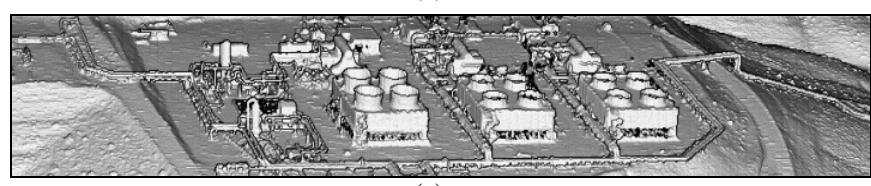

(c)

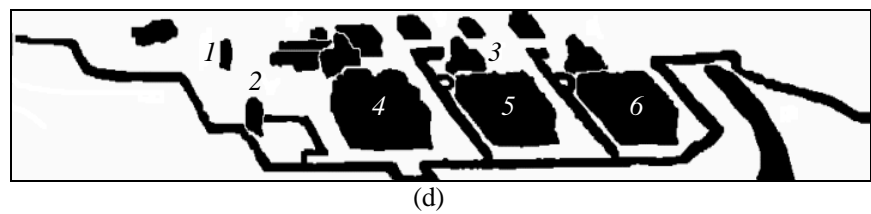

Fig. 10. (a) Filtered LADAR range image of a Surface-to-Air Missile site with convoy. (b) Objects detected by the Automatic Target Recognition system described in [20]. (c) Filtered LADAR range image of a power plant. (d) Hand-segmented image used in [8].

the spatial relationship which suits the configuration best. This relationship is also shown in Fig. 11. In Fig. 11(g), the fuzzy system presented in [8] detects a large amount of ambiguity. It states that " $A$ is loosely below $B$ " and notes that "the description is unsatisfactory." The second part of the message prompts the user to turn to relationships other than directional ones. The extended system would stick to this output anyhow, because the values $s(A, B), b(A, B)$ and $a(A, B)$ are too low. In Fig. 11(j), the amount of ambiguity detected by the original system is even larger, and no appropriate description can be given. Although the value $s(A, B)$ is quite high, one should not state that " $A$ is surrounded by $B$." As happens with the rulebased methods (Section II.B), it is important to make sure beforehand that $B$ does not intersect the convex hull of $A$ (or that $A$ is "rather" compact, and that $A$ and $B$ do not intersect "much")—which is not the case here. This is the price to pay for concurrent assessment of directional relationships and spatial relationships like "surrounded by," "between" and "among." Finally, here are some results obtained when choosing other basic probability assignments (see Section III.D). With the standard assignments (Equation (4)), the highest degrees of truth are often lower than 0.3 , and no relationship really stands out. When $f$ is the steep L-shaped function (the $\alpha$-cuts of the fuzzy set $H$ are ignored, only the support $H^{0}$ is considered), the results tend to be crisp, and are much less satisfactory (according to our own perception). In Fig. 11(d) for instance, $A$ is found to be "between $B$ " $(b(A, B)=0.85$ and $a(A, B)=0.72)$; for Fig. $11(\mathrm{~g})$, we get $s(A, B)=0.91$ (instead of 0.41); in Fig. 11(h), $A$ is "surrounded by $B$ " $(s(A, B)=0.94$ and $b(A, B)=0.00)$; in Fig. 11(i), we have $s(A, B)=1.00$ (instead of 0.63). 


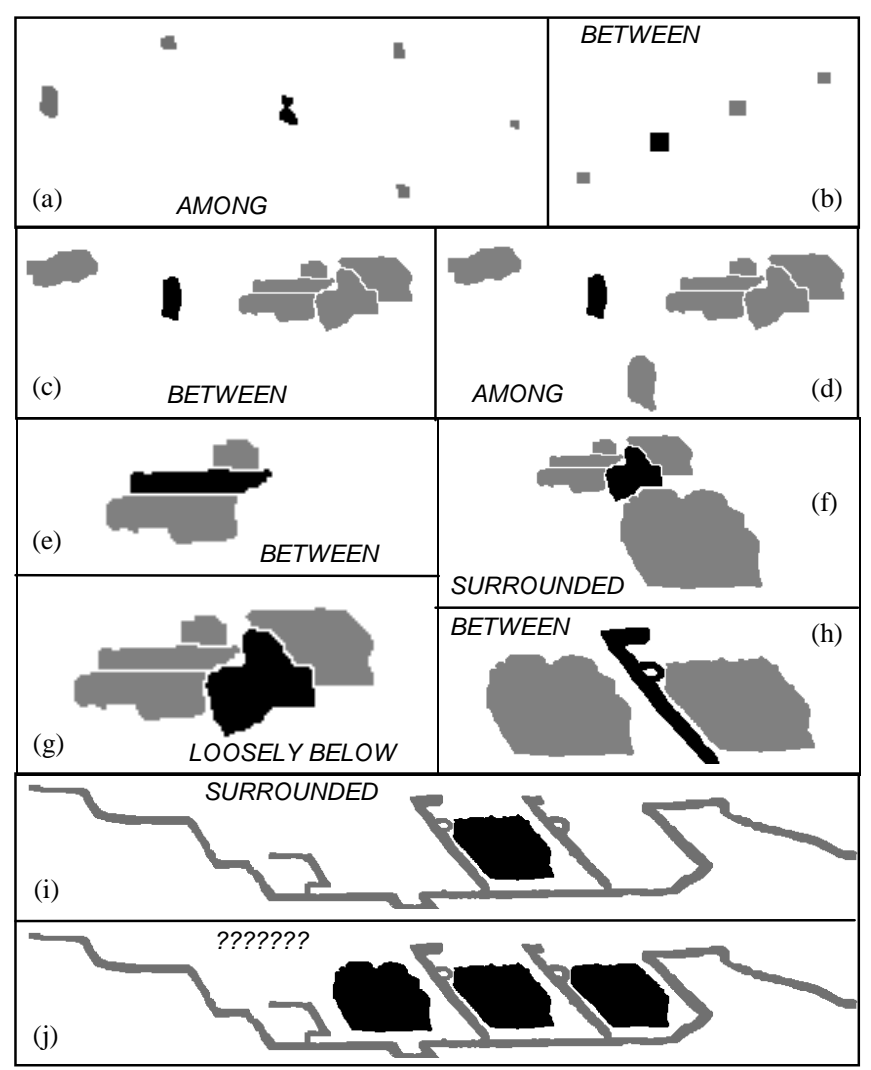

Fig. 11. Ten configurations extracted from the images shown in Fig. 10.. $A$ is the black object, $B$ is the union of the gray ones.

TABLE II

VALUES OF $s(A, B), b(A, B)$ AND $a(A, B)$ FOR THE CONFIGURATIONS DEPICTED IN FIG. 11

\begin{tabular}{|c|c|c|c|c|c|c|c|c|c|c|}
\cline { 2 - 12 } \multicolumn{1}{c|}{} & (a) & (b) & (c) & (d) & (e) & (f) & (g) & (h) & (i) & (j) \\
\hline$s$ & 0.23 & 0.08 & 0.07 & 0.23 & 0.58 & $\mathbf{0 . 9 2}$ & 0.41 & 0.58 & $\mathbf{0 . 6 3}$ & 0.68 \\
\hline$b$ & 0.29 & $\mathbf{0 . 9 2}$ & $\mathbf{0 . 7 1}$ & 0.47 & $\boldsymbol{0 . 6 2}$ & 0.02 & 0.01 & $\mathbf{0 . 6 1}$ & 0.16 & 0.12 \\
\hline$a$ & $\mathbf{0 . 7 1}$ & 0.00 & 0.00 & $\mathbf{0 . 6 7}$ & 0.06 & 0.02 & 0.01 & 0.00 & 0.00 & 0.00 \\
\hline
\end{tabular}

\section{CONCLUSION}

We have shown that the histogram of forces can be employed to design consistent fuzzy models of spatial relationships like "surrounded by," "between" and "among." Compared to other existing methods, force histogram-based methods are computationally much less expensive; they are able to handle non-connected objects and groups of objects; they are able to handle vector data as well as raster data; and a variety of spatial models, more or less sensitive to the thickness of the objects and to the distance between them, can be defined. Another important advantage is that the directional relations (i.e., "to the left of," "above," etc.) can be assessed concurrently. Unfortunately, there is a price to pay: as happens with the rule-based methods, we have to make sure beforehand that the considered objects satisfy certain constraints (e.g., one object is compact and does not intersect the other object, or one of the objects does not intersect the convex hull of the other object). Only specific tools, dedi- cated to the evaluation of the spatial relationships studied here, can overcome this limitation. Designing new types of force histograms constitutes a promising avenue. The idea is to adopt novel sets of axiomatic properties, and to change the way the longitudinal sections are handled [4][5].

\section{ACKNOWLEDGEMENTS}

The authors want to express their gratitude for support from the Office of Naval Research grant N00014-96-0439, and to the personnel of the Naval Air Warfare Center for their assistance with the LADAR imagery. They also wish to thank Marzena Puzniak for her precious advice and comments.

\section{REFERENCES}

[1] J. Freeman, "The Modeling of Spatial Relations", Computer Graphics and Image Processing, vol. 4, pp. 156-171, 1975.

[2] R. Krishnapuram, J. M. Keller and Y. Ma, "Quantitative Analysis of Properties and Spatial Relations of Fuzzy Image Regions", IEEE Trans. on Fuzzy Systems, vol. 1, no. 3, pp. 222-233, 1993.

[3] K. Miyajima and A. Ralescu, "Spatial Organization in 2D Segmented Images: Representation and Recognition of Primitive Spatial Relations", Fuzzy Sets and Systems, vol. 65, iss. 2/3, pp. 225-236, 1994.

[4] P. Matsakis, Relations spatiales structurelles et interprétation d'images, Ph.D. Thesis, Institut de Recherche en Informatique de Toulouse, France, 1998.

[5] P. Matsakis and L. Wendling, "A New Way to Represent the Relative Position between Areal Objects," IEEE Trans. on Pattern Analysis and Machine Intelligence, vol. 21, no. 7, pp. 634-643, 1999.

[6] P. Matsakis and L. Wendling, "Orbit and Sinus Classification based on Force Histogram Computation," ICPR'2000 (Int. Conf. on Pattern Recognition), Barcelona, Spain, Proceedings, vol. 2, pp. 451-54.

[7] P. Matsakis, J. Keller, O. Sjahputera and J. Marjamaa, "Matching Image Object Pairs while Retrieving the Pose Parameters," PAMI (IEEE Pattern Analysis and Machine Intelligence), submitted.

[8] P. Matsakis, J. Keller, L. Wendling, J. Marjamaa and O. Sjahputera, "Linguistic Description of Relative Positions in Images," TSMC Part B (IEEE Trans. on Systems, Man and Cybernetics), vol. 31, no. 4, pp. 573-588, 2001.

[9] C. Shyu and P. Matsakis, "Spatial Lesion Indexing for Medical Image Databases Using Force Histograms," CVPR' 2001(Int. Conf. on Computer Vision and Pattern Recognition), Hawaii, Proceedings, to appear.

[10] R. Krishnapuram and J. Keller, "Fuzzy Set Theoretic Approach to Computer Vision: An Overview," FUZZ-IEEE'1992, New York, USA, Proceedings, pp. 135-42.

[11] K. Miyajima and A. Ralescu, "Spatial Organization in 2D Segmented Images," FUZZ-IEEE'1994, Orlando, FL, Proceedings, pp. 100-105.

[12] G. Retz-Schmidt, "Various Views on Spatial Prepositions," AI Magazine, vol. 9, no. 2, pp. 95-105, 1988.

[13] K. P. Gapp, "Angle, Distance, Shape, and their Relationship to Projective Relations," Conf. of the Cognitive Science Society, 1995, Proceedings.

[14] X. Wang and J. M. Keller, "Fuzzy surroundedness," FUZZ-IEEE'1997, Proceedings, vol. 2, pp. 1173-8.

[15] A. Rosenfeld and R. Klette, "Degree of Adjacency or Surroundedness", Pattern Recognition, vol. 18, no. 2, pp. 167-77, 1985.

[16] J. M. Keller and X. Wang, "Comparison of Spatial Relation Definitions in Computer Vision," ISUMA-NAFIPS'95, College Park MD, Proceedings, pp. 679-684.

[17] D. Dubois and M.-C. Jaulent, "A General Approach to Parameter Evaluation in Fuzzy Digital Pictures," Pattern Recognition Letters, vol. 6, pp. 251-259, 1987.

[18] G. Shafer, A Mathematical Theory of Evidence, Princeton University Press, Princeton, NJ, 1976.

[19] L. Zadeh, "Fuzzy Sets as Basis for a Theory of Possibility," Fuzzy Sets and Systems, vol. 1, pp. 3-28, 1976.

[20] J. Keller, P. Gader, R. Krishnapuram, X. Wang, K. Hocaoglu, H. Frigui, J. Moore, "Fuzzy Logic Automatic Target Recognition System For LADAR Range Images,' FUZZ-IEEE'1998, Anchorage, Proceedings, pp. 71-76. 